\title{
Extracting Double Diode Model Parameters based on Cross Entropy Optimization Algorithm
}

\author{
Latifa Sabri ${ }^{\star}$, Mohammed Benzirar, Mimoun Zazoui \\ Laboratory of Physics of Condensed Matter and Renewable Energy, Faculty of Sciences and Technology, \\ Hassan II University of Casablanca, BP 146, Mohammedia, Morocco \\ ${ }^{*}$ Corresponding author, e-mail: sabri.latifa@gmail.com
}

\begin{abstract}
A Cross Entropy (CE) optimization technique is suggested in this study in order to extract the optimal parameters of solar cell model. The solar cell model namely Double diode model, is used in this paper in the purpose to verify the proposed method. The seven unknown parameters of double diode model are: the photo-generated current, the diffusion current, saturation current, the series resistance, the shunt resistance, the diffusion ideality factor and recombination diode ideality factor. These parameters were used to determine the corresponding maximum power point (MPP) from the illuminated currentvoltage $(I-V)$ characteristic. The metaheuristic algorithms have created a center of attention due to the non-linearity of the solar cell models and the incapability of traditional optimization methods to precisely extract the parameters. Different cases and studies have been done to identify the unknown parameters of the solar cell double model. The efficiency of CE algorithm is investigated by comparing this method with various and different other techniques. This comparison is done using statistical indicators and analysis such as: mean absolute bias error, root mean square error and coefficient of determination. The results show that $C E$ is a very efficient technique to identify the electrical parameters of the PV solar cells and modules.
\end{abstract}

Keywords: parameter estimation, photovoltaic, solar cell, cross entropy, double diode model

\section{Introduction}

The world now knows a decline of fossil fuels for energy production. As a solution to this problem, there is an option of solar energy based on photovoltaic cells. This PV (Photovoltaic) system uses solar energy using solar cells without emitting pollutants and requiring no fuel.

Different models have been suggested to explain the Current-voltage relationship (I-V) in [1-3]. The I-V curve of solar cell shows non-linear characteristics determined by the solar cell parameters that describe its model. A lumped parameter equivalent circuit model is commonly used to simulate the behavior the solar cell under different operating conditions in order to gain better understanding of the solar cell physics. In practice, two main equivalent circuit models that are used to describe the I-V relationship: single and double diode models. But this paper will just focus on the double diode model. The key parameters that describe the double diode model behavior are: the photo-generated current, the diffusion current, saturation current, the series resistance, the shunt resistance, the diffusion ideality factor and recombination diode ideality factor. A perfect estimation of these parameters is always required to provide precise modeling and accurate performance evaluation of a given solar system.

Various studies have used different techniques and approaches in order to estimate different parameters of solar cells. Two main approaches have been used in the literature to solve the parameter identification problem: traditional techniques [4-6] and metaheuristic approaches like: Global Grouping- based Harmony Search (GGHS) [7], Artificial Bee Swarm Optimization (ABSO) [8], Chaotic Particle Swarm Optimization (CPSO) [9], Differential Evolution (DE) [10], Pattern Search (PS) [11], Simulated Annealing (SA) [12] and Newton-Raphson [13]. The high accuracy of CE shows its ability and its efficiency to identify the most accurate values of the model parameters of commercial solar cell (R.T.C. France), based on experimental data.

The paper is structured as follows. Section 2 describes the solar cell modeling. In Section 3, describes the CE algorithm proposed in this paper in detail. While Section 4 describes in detail several statistical indicators used to quantify the performance accuracy results of the prediction models with the provided measurements. Thereafter, Section 5 
presents the obtained results and the related discussions, while the Section 6 gives a conclusion of the paper.

\section{Problem Formulation}

\subsection{Double Diode Model}

The solar cell is, ideally, modeled under illumination as a current source connected in parallel with a rectifying diode. But, the current source is also shunted by another diode in order to consider the space charge recombination current and a shunt leakage resistor to take into account the partial short circuit current path near the cell's edges related to the semiconductor impurities and non-idealities. Moreover, a series resistor is connected with the cell shunt elements because of the solar cell metal contacts and the semiconductor material bulk resistance [14].The equivalent circuit of a double diode model is presented in Figure 1.

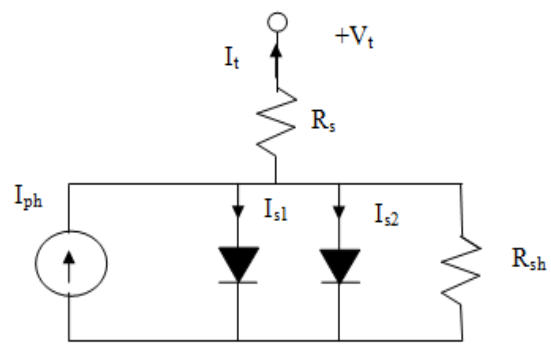

Figure 1. Equivalent circuit of a double diode model

The terminal current that allows the calculation of the $I_{t}$ is obtained as follows:

$$
I_{t}=I_{p h}-I_{S 1}\left[\exp \left(\frac{q\left(V_{t}+I_{t} R_{S}\right)}{n_{1} K T}\right)-1\right]-I_{S 2}\left[\exp \left(\frac{q\left(V_{t}+I_{t} R_{S}\right)}{n_{2} K T}\right)-1\right]-\frac{V_{t}+I_{t} R_{S}}{R_{S h}}
$$

Where, $I_{p h}$ is the photo-generated current, $I_{S 1}$ and $I_{S 2}$ are the diffusion and saturation currents, respectively, $V_{t}$ is the terminal voltage, $R_{s}$ and $R_{s h}$ are the series and shunt resistances, $q$ is the electronic charge, $K$ denotes the Boltzmann constant, $n_{1}$ and $n_{2}$ are the diffusion and recombination diode ideality factors, and $T$ is the cell temperature.

From this equation, there are seven parameters to be extracted which are: $I_{p h}, I_{S 1}, I_{S 2}$, $R_{s h}, n_{1}$, and $n_{2}$. This objective is done by using an optimization method and an experimental data that are obtained from the real system.

We can write $f(x)=I_{t}$ with $x$ is the vector of the seven parameters $\left(I_{p h}, I_{S 1}, I_{S 2}, R_{s h}, n_{1}, n_{2}\right.$.).

\subsection{Optimization Process}

An objective function or a performance criterion should be first defined before proceeding to the optimization stage. This paper proposed an absolute error objective function to minimize the Sum of Squared Error (SSE), for any given set of measurements which is given as:

$$
F(x)=\sum_{i=1}^{n}\left(I_{t, m i}-f_{i}(x)\right)^{2}
$$

Where $\mathrm{N}$ is the number of data points $I_{t, m i}$ is ith measured current values. In the optimization algorithm, each solution is defined by a vector, $\mathrm{x}$, where $\mathrm{x}=\left[R_{s}, R_{s h}, I_{p h}, I_{s 1}, I_{s 2}, n_{1}, n_{2}\right]$.

During the CE (Cross Entropy) optimization process, the objective function is to be minimized with respect to the parameter set. Theoretically, the objective function should have zero value when the parameters' exact values are obtained. In other words, the objective function should be zero for any experimental set of I-V data when the exact value has been determined for each parameter. However, it is expected to obtain a very small non zero value due to the presence of measuring noise errors. Moreover the smaller the objective function, the better the solution obtained. 


\section{Cross Entropy Method (CE)}

Introduced by Rubinstein, the cross-entropy (CE) method is a generic Monte Carlo for solving complicated estimation and optimization problems [15]. The CE method can be used for two types of problems: estimation or optimization problems (minimize $\mathrm{F}(\mathrm{x})$ (like in our case) or maximize), where $F$ is an objective function on $x$ and it can be applied to different fields.

The CE method has been successfully applied to a variety of problems in combinatorial optimization and rare event estimation, the latter with both light and heavy tailed distributions. Applications areas include buffer allocation, queuing models of telecommunication systems, neural computation, control and navigation, DNA sequence alignment, signal processing, scheduling, vehicle routing, reinforcement learning, project management and reliability systems. It is important to note that the CE method is developed as an efficient method for the estimation of rare event probabilities.

The CE method has been successfully applied to a number of difficult combinatorial optimization problems. More details and explanation on applications and theory can be found in the CE tutorial [16], the recent CE monograph [17] and [18].

The CE method consists of two phases:

a. Generate a random data sample (trajectories, vectors, etc.) according to a specified mechanism.

b. Update the parameters of the random mechanism based on the data to produce a "better" sample in the next iteration.

The CE method is based on the generation of a random data sample $(x 1 \ldots x N)$ according to a specified random mechanism. A reasonable option is to use a probability density function (pdf) such as the normal distribution

$g(., v)$.

So the aim of the CE method is to find the minimum (like in our case) or maximum of $F$, and the corresponding parameters $x^{*}$ satisfying this minimum/maximum:

$$
y^{*}=F\left(x^{*}\right)=\min _{x \varepsilon X} F(x)
$$

In each iteration, the CE method generates a sequence of $(x 1, \ldots, x N)$. The CE algorithm generates a set of $N$ samples $x i$ and calculates the performance function value for each sampler. The parameters $x 1, x 2, x 3, x 4, x 5, x 6$ and $x 7$ correspond to the gains of the first, second, third, fourth, fifth, sixth and seventh input of each sampler. Then updates $g(x, v)$ using a set of the best samples. This set of samples is defined with the parameter $\mathrm{N}$ elite. The process finish when the minimum value of the performance function or the maximum number of iterations is reached, as is shown and presented step by step in Figure 2. The convergence procedure of $C E$ is stopped when the solution is reached.

For this work, the mean mu is estimated to $m u=[0.03,50,2,0.3,1.5,0.4,0.76]$ and standard deviation is estimated as ones $(1, n)$ where $n$ is the number of parameters which is equal to 7 in this case. 


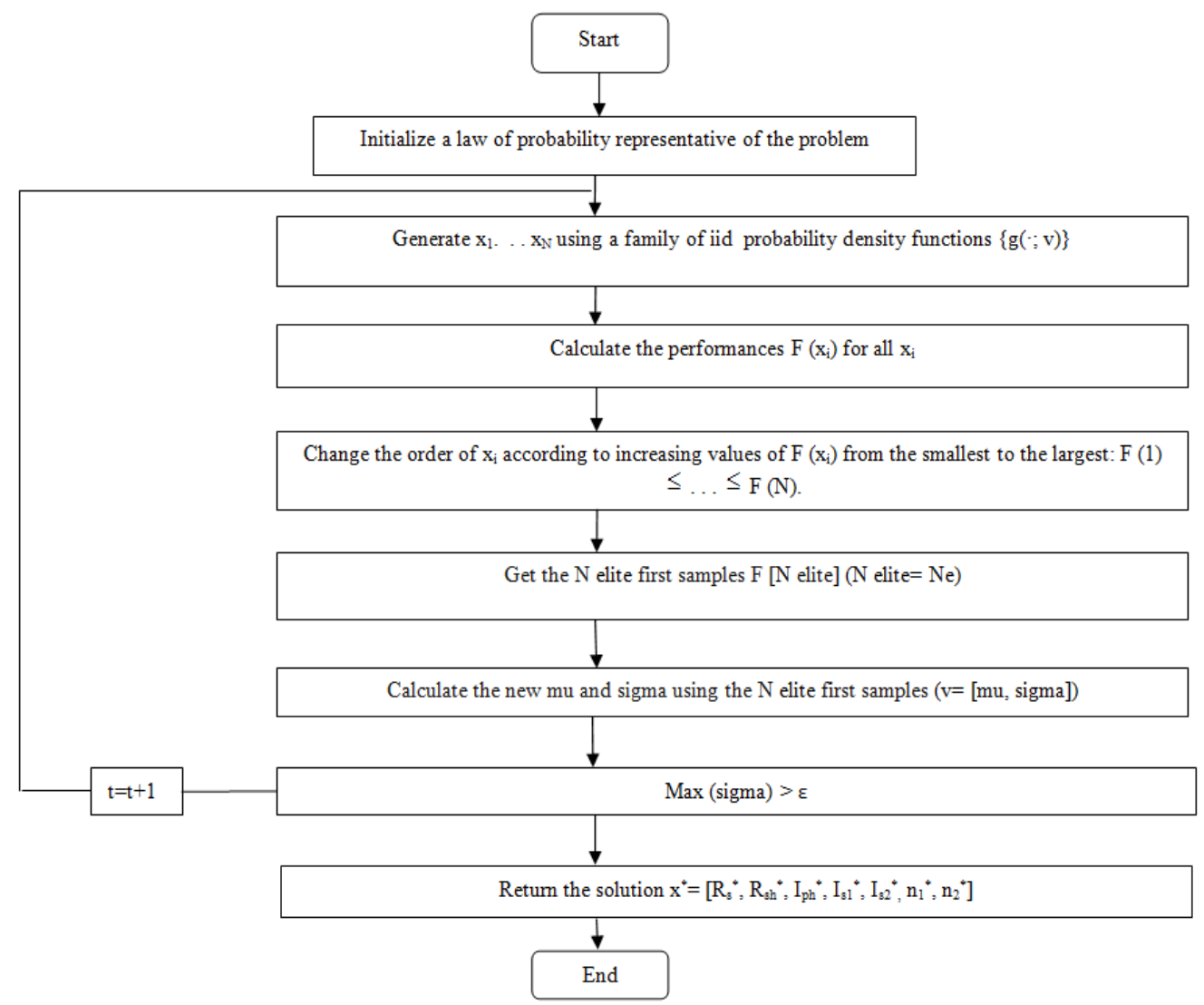

Figure 2: Flowchart of CE algorithm

\section{Statistical Performance Validation}

Performance of the used methods has been evaluated based on the following well established statistical error parameters of MBE, RMSE, MABE, Coefficient of Determination $R^{2}$ and MAE (dimensionless). All these indicators are represented in Equations (4-8), which were used to judge the validity of the models.

The smaller values of MBE, RMSE, MABE and MAE show more validity of the prediction values. The $R^{2}$ takes values between 0 and 1.A value around 1 means perfect linear relationship between the estimated and calculated values. While a value around zero indicates the absence of the linear relationship. These statistical indicators tell us whether these algorithms are statistically significant or not.

Mean Bias Error (MBE) defined as:

$$
M B E=\frac{\Sigma I_{m}-I_{C}}{N}
$$

Root Mean Square Error (RMSE) defined as:

$$
R M S E=\left(\frac{\Sigma\left(I_{m}-I_{c}\right)^{2}}{N}\right)^{\frac{1}{2}}
$$

Mean Absolute Bias Error (MABE) defined as

$$
M A B E=\frac{\Sigma\left|I_{c}-I_{m}\right|}{N}
$$

Coefficient of determination R2 defined as: 


$$
R^{2}=1-\frac{\Sigma\left(I_{m}-I_{c}\right)^{2}}{\Sigma\left(I_{m}-I_{M}\right)^{2}}
$$

Mean Absolute Error (MAE) defined as:

$$
M A E=\frac{1}{N} \sum\left|\frac{I_{c}-I_{m}}{I_{m}}\right|
$$

where $\mathrm{N}$ is the total number of observations, $I_{m}$ is measured data, $I_{c}$ are calculated, $I_{M}$ is the mean of the measured data.

\section{Results and Discussion}

A $57 \mathrm{~mm}$ diameter commercial (R.T.C. France) silicon solar cell is considered in order to study the efficiency of CE algorithm in extracting and identifying the seven unknown parameters. The experimental data from the system under 1 sun $\left(1000 \mathrm{~W} / \mathrm{m}^{2}\right)$ at $33^{\circ} \mathrm{C}$ [13] has been adopted. This experimental data is shown in Table 1.

\begin{tabular}{ccc}
\multicolumn{3}{c}{ Table 1. Experimental Recorded Data } \\
\hline Measurement & $\mathrm{Vt}(\mathrm{V})$ & It measured $(\mathrm{A})$ \\
\hline 1 & -0.2057 & 0.764 \\
2 & -0.1291 & 0.762 \\
3 & -0.0588 & 0.7605 \\
4 & 0.0057 & 0.7606 \\
5 & 0.0646 & 0.76 \\
6 & 0.1185 & 0.759 \\
7 & 0.1678 & 0.757 \\
8 & 0.2132 & 0.7571 \\
9 & 0.2545 & 0.7555 \\
10 & 0.2924 & 0.754 \\
11 & 0.3269 & 0.7505 \\
12 & 0.3585 & 0.7465 \\
13 & 0.3873 & 0.7385 \\
14 & 0.4137 & 0.728 \\
15 & 0.4373 & 0.7065 \\
16 & 0.459 & 0.6755 \\
17 & 0.4784 & 0.632 \\
18 & 0.496 & 0.573 \\
19 & 0.5119 & 0.499 \\
20 & 0.5265 & 0.413 \\
21 & 0.5398 & 0.3165 \\
22 & 0.5521 & 0.212 \\
23 & 0.5633 & 0.1035 \\
24 & 0.5736 & -0.01 \\
25 & 0.5833 & -0.123 \\
26 & 0.59 & -0.21 \\
\hline & &
\end{tabular}

The algorithm is coded and executed in the MATLAB environment to extract the double diode model parameters. The optimal parameters for double diode model found by CE method is summarized in Table 2. WhileTable 3 shows the results of optimal parameters obtained by CE for double diode model in comparison with the results found by artificial bee swarm optimization (ABSO)[8],pattern search (PS) [11] ,harmony search based algorithms HS [7] and simulated annealing (SA) [12].Based on the extracted parameters, the I-V data set is reconstructed.

Table 4 compared the obtained IAE values for the double diode model with those obtained using three other techniques in [8], [13] and [11]. While Table 5 shows the resultant MAE for each data point for the double diode model.

Figures 3 and 4 shows the I-V and P-V characteristics for the double diode model based on the extracted parameters using CE method. The calculated values are accordance with the measured data observably. It shows good agreement for most of the extracted parameters. 
Table 2 :Estimated Parameters for the Double Diode Model

\begin{tabular}{cc}
\hline Parameter & CE \\
\hline Rs $(\Omega)$ & 0.026 \\
Rsh $(\Omega)$ & 49.9679 \\
Iph $(A)$ & 0.7666 \\
Is1 $(\mu \mathrm{A})$ & 0.2932 \\
Is2 $(\mu \mathrm{A})$ & 1.49607 \\
$\mathrm{n} 1$ & 1.5068 \\
$\mathrm{n} 2$ & 1.9992 \\
\hline
\end{tabular}

Table 3 : Results Obtained by CE and the Other Methods for Double Diode Model

\begin{tabular}{cccccc}
\hline & CE & ABSO & PS & HS & SA \\
\hline Rs $(\Omega)$ & 0.026 & 0.03657 & 0.0320 & 0.03545 & 0.0345 \\
Rsh $(\Omega)$ & 49.9679 & 54.6219 & 81.3008 & 46.8269 & 43.1034 \\
Iph $(A)$ & 0.7666 & 0.76078 & 0.7602 & 0.76176 & 0.7623 \\
Is1 $(\mu \mathrm{A})$ & 0.2932 & 0.26713 & 0.9889 & 0.12545 & 0.4767 \\
Is2 $(\mu \mathrm{A})$ & 1.49607 & 0.38191 & 0.0001 & 0.25470 & 0.0100 \\
n1 & 1.5068 & 1.46512 & 1.6000 & 1.49439 & 1.5172 \\
n2 & 1.9992 & 1.98152 & 1.1920 & 1.49989 & 2.0000
\end{tabular}

Table 4: IAEs Based on the Extracted Parameters for Double Diode Model

\begin{tabular}{|c|c|c|c|c|c|c|}
\hline Measurement & $\mathrm{Vt}(\mathrm{V})$ & $\begin{array}{c}\text { It } \\
\text { measured }(A)\end{array}$ & $\begin{array}{c}\text { IAE } \\
\text { based on } \\
\text { CE }\end{array}$ & $\begin{array}{c}\text { IAE based } \\
\text { on } \\
\text { ABSO }\end{array}$ & $\begin{array}{c}\text { IAE } \\
\text { based } \\
\text { on PS }\end{array}$ & $\begin{array}{c}\text { IAE based } \\
\text { on HS }\end{array}$ \\
\hline 1 & -0.2057 & 0.764 & 0.00632 & 0.00020 & 0.00158 & 0.00025 \\
\hline 2 & -0.1291 & 0.762 & 0.00679 & 0.00736 & 0.00053 & 0.00081 \\
\hline 3 & -0.0588 & 0.7605 & 0.00688 & 0.00089 & 0.00011 & 0.00086 \\
\hline 4 & 0.0057 & 0.7606 & 0.00549 & 000034 & 0.00069 & 0.00023 \\
\hline 5 & 0.0646 & 0.76 & 0.00491 & 0.00097 & 0.00092 & 0.00084 \\
\hline 6 & 0.1185 & 0.759 & 0.00482 & 0.00101 & 0.00061 & 0.00087 \\
\hline 7 & 0.1678 & 0.757 & 0.00580 & 0.00002 & 0.00072 & 0.00017 \\
\hline 8 & 0.2132 & 0.7571 & 0.00469 & 0.00096 & 0.00002 & 0.00079 \\
\hline 9 & 0.2545 & 0.7555 & 0.00520 & 0.00053 & 0.00053 & 0.00035 \\
\hline 10 & 0.2924 & 0.754 & 0.00530 & 0.00046 & 0.00053 & 0.00029 \\
\hline 11 & 0.3269 & 0.7505 & 0.00670 & 0.00078 & 0.00141 & 0.00093 \\
\hline 12 & 0.3585 & 0.7465 & 0.00713 & 0.00076 & 0.00067 & 0.00088 \\
\hline 13 & 0.3873 & 0.7385 & 0.00889 & 0.00156 & 0.00033 & 0.00163 \\
\hline 14 & 0.4137 & 0.728 & 0.00859 & 0.00061 & 0.00336 & 0.00061 \\
\hline 15 & 0.4373 & 0.7065 & 0.01282 & 0.00054 & 0.00382 & 0.00048 \\
\hline 16 & 0.459 & 0.6755 & 0.01686 & 0.00010 & 0.00585 & 0.00020 \\
\hline 17 & 0.4784 & 0.632 & 0.02196 & 0.00111 & 0.00753 & 0.00120 \\
\hline 18 & 0.496 & 0.573 & 0.02899 & 0.00096 & 0.00710 & 0.00099 \\
\hline 19 & 0.5119 & 0.499 & 0.03699 & 0.00016 & 0.00427 & 0.00073 \\
\hline 20 & 0.5265 & 0.413 & 0.04153 & 0.00243 & 0.00232 & 0.00083 \\
\hline 21 & 0.5398 & 0.3165 & 0.04271 & 0.00018 & 0.00019 & 0.00123 \\
\hline 22 & 0.5521 & 0.212 & 0.03760 & 0.00016 & 0.00130 & 0.00039 \\
\hline 23 & 0.5633 & 0.1035 & 0.02570 & 0.00155 & 0.00112 & 0.00102 \\
\hline 24 & 0.5736 & -0.01 & 0.01377 & 0.00103 & 0.00343 & 0.00146 \\
\hline 25 & 0.5833 & -0.123 & 0.01878 & 0.00272 & 0.00158 & 0.00241 \\
\hline 26 & 0.59 & -0.21 & 0.03922 & 0.00156 & 0.00007 & 0.00153 \\
\hline Total IAE & & & 0.42444 & 0.02895 & 0.05059 & 0.02200 \\
\hline
\end{tabular}

Table 5: Mean Absolute Error (MAE) Based on the Extracted Parameters (Double Diode Model)

\begin{tabular}{ccccc}
\hline Measurement & $\mathrm{Vt}(\mathrm{V})$ & It measured $(\mathrm{A})$ & It calculated $(\mathrm{A})$ & Relative error \\
\hline 1 & -0.2057 & 0.764 & 0.77032 & -0.00827197 \\
2 & -0.1291 & 0.762 & 0.76879 & -0.00890786 \\
3 & -0.0588 & 0.7605 & 0.76738 & -0.00904857 \\
4 & 0.0057 & 0.7606 & 0.76609 & -0.00721751 \\
5 & 0.0646 & 0.76 & 0.76491 & -0.00645766 \\
6 & 0.1185 & 0.759 & 0.76382 & -0.00634818 \\
7 & 0.1678 & 0.757 & 0.76280 & -0.00765857 \\
8 & 0.2132 & 0.7571 & 0.76179 & -0.00619197 \\
9 & 0.2545 & 0.7555 & 0.76070 & -0.00688201 \\
10 & 0.2924 & 0.754 & 0.75930 & -0.00703488 \\
11 & 0.3269 & 0.7505 & 0.75720 & -0.00892751 \\
12 & 0.3585 & 0.7465 & 0.75363 & -0.00954742
\end{tabular}




\begin{tabular}{ccccc}
\hline Measurement & $\mathrm{Vt}(\mathrm{V})$ & It measured $(\mathrm{A})$ & It calculated $(\mathrm{A})$ & Relative error \\
\hline 13 & 0.3873 & 0.7385 & 0.74739 & -0.01204386 \\
14 & 0.4137 & 0.728 & 0.73659 & -0.01180341 \\
15 & 0.4373 & 0.7065 & 0.71932 & -0.01814386 \\
16 & 0.459 & 0.6755 & 0.69236 & -0.0249633 \\
17 & 0.4784 & 0.632 & 0.65396 & -0.03474427 \\
18 & 0.496 & 0.573 & 0.60199 & -0.05059777 \\
19 & 0.5119 & 0.499 & 0.53599 & -0.07412701 \\
20 & 0.5265 & 0.413 & 0.45453 & -0.1005569 \\
21 & 0.5398 & 0.3165 & 0.35921 & -0.13495115 \\
22 & 0.5521 & 0.212 & 0.24960 & -0.17734457 \\
23 & 0.5633 & 0.1035 & 0.12920 & -0.24831622 \\
24 & 0.5736 & -0.01 & 0.00423 & 0.76512826 \\
25 & 0.5833 & -0.123 & -0.14178 & -0.1526666 \\
26 & 0.59 & -0.21 & -0.24922 & -0.1867403 \\
\hline
\end{tabular}

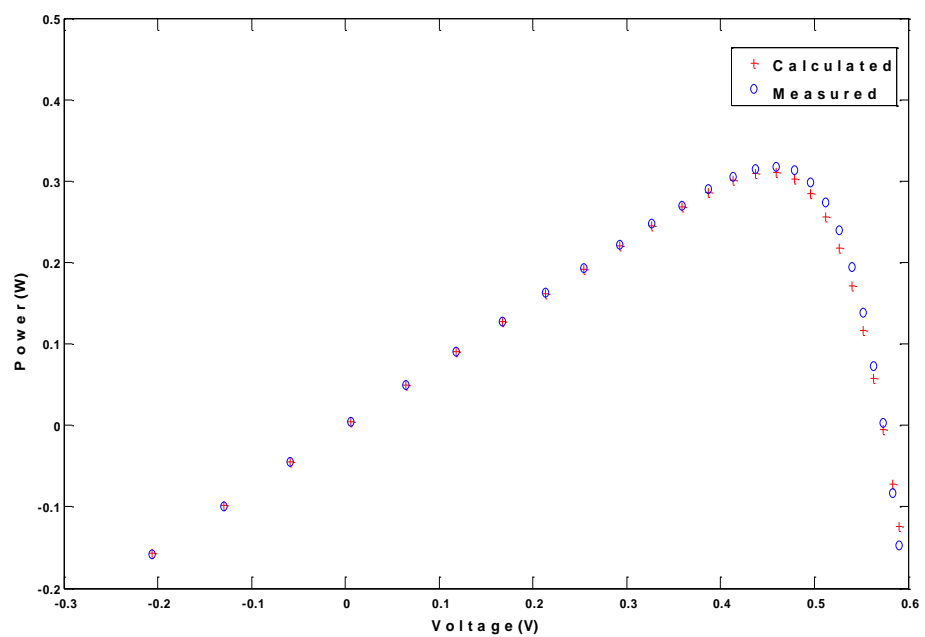

Figure 3. I-V characteristics resulted from the experimental data and the double diode model

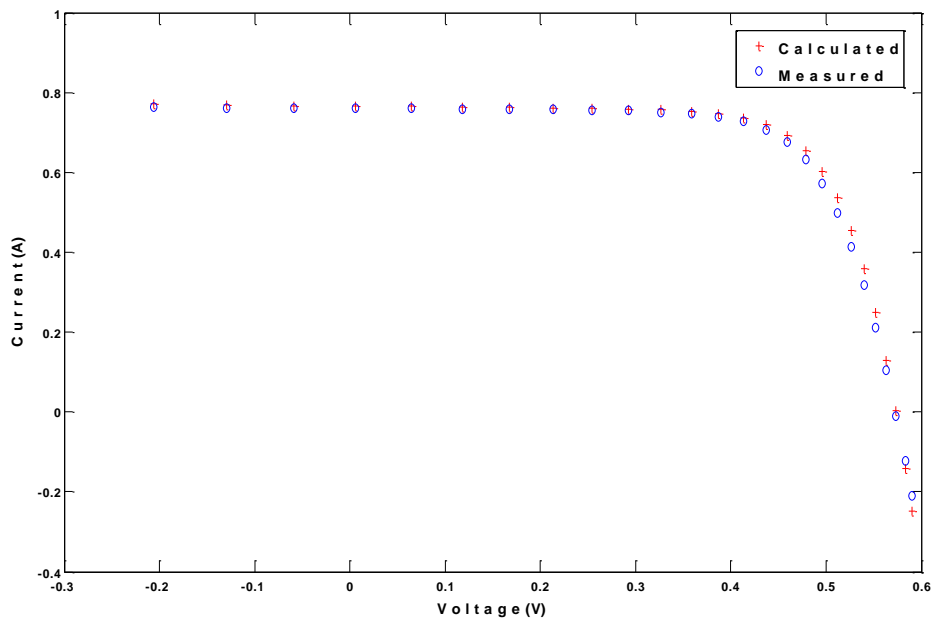

Figure 4. P-V characteristics resulted from the experimental data and the double diode model

The statistical parameter values obtained of MBE, RMSE, MABE and coefficient of determination R2 are presented in Table 6 . In terms of the statistical analysis, it is observed from Table 6 that the presented models in this study perform several results for considered methods. Basing on the values of statistical indicators, the coefficient of determination R2 
presents the perfect linear relationship between the measured and computed values for all methods, where it is always closed to 1 value for CE, ABSO, HS, PS and SA.

Table 6. A Comparisons Between the Results Obtained by the CE Algorithm and the Other Ones for the Double Diode Model Parameter Identification

\begin{tabular}{cccccc}
\hline & CE & ABSO & PS & HS & SA \\
\hline RMSE & 0.02095 & 0.00187 & 0.00288 & 0.00187 & 0.00183 \\
MBE & 0.01186 & 0.00030 & -0.00195 & 0.00030 & -0.00138 \\
MABE & 0.01632 & 0.00111 & 0.00195 & 0.00085 & 0.00138 \\
R2 & 0.995097019 & 0.999961525 & 0.999906745 & 0.999960979 & 0.999963215 \\
\hline
\end{tabular}

The summation of the IAE obtained using the parameters estimated via CE technique is 0.42444 as shown in Table 4. A reduction in IAE summation is clear when CE results are compared with other reported outcomes which are $0.02895,0.05059$ and 0.02200 respectively for ABSO, PS and HS.

These lower values also indicate the best performance of these methods, where they present the differences between estimated and measured values. The MABE values are, in generally low, and it found 0.01632 for CE method, 0.00111 for ABSO method, 0.00195 for PS method, 0.00085 for HS method and 0.00138 for SA method, this also signify better performances. Finally as showed results previously and as it appears in Figs. 3 and 4 and Tables 3 and 4, it can conclude, that CE method can be employed to extract the parameters of double diode model.

In order to confirm the quality of the fit to experimental data, the statistical analysis based on mean absolute error (MAE) is made. Table 5 shows the relative error, for each measurement along with the MAE. The calculated value of the MAE denotes the high accuracy of the identification process.

\section{Conclusion}

The CE method is proposed in this paper for parameter identification of the solar cell mathematical model, namely, the double diode model. The main objective of this study is to identify the seven unknown parameters for the double diode model in order to acquire an accurate I-V characteristic of a real system that is composed of a $57 \mathrm{~mm}$ diameter commercial (R.T.C. France) silicon solar cell. The obtained results using the CE algorithm especially when compared to other methods are quite promising and deserve serious attention. As well; the outcomes are examined using the statistical indicators such as: Coefficient of Determination $\mathrm{R}^{2}$, Mean Bias Error (MBE), Root Mean Square Error (RMSE), Mean Absolute Bias Error (MABE) and Mean Absolute Error (MAE) to show their accuracy. As a result, the CE technique is a good and capable approach that can be efficiently applied to solve the optimization problems of solar cell model.

\section{References}

[1] Chegaar M, Ouennoughi Z, Guechi F, Langueur H. Determination of Solar Cells Parameters under Illuminated Conditions. Journal of Electron Devices. 2003; 2:17-21.

[2] Xiao W, Lind MGJ, Dunford WG, Capel A. Real-time identification of optimal operating points in photovoltaic power systems. IEEE Transactions on Industrial Electronics. 2006; 53:1017-1026.

[3] Ye M, Wang X, Xu Y. Parameter extraction of solar cells using particle swarm optimization. Journal of Applied Physics. 2009; 105: 0-8.

[4] Jain A, Kapoor A. Exact analytical solutions of the parameters of real solar cells using Lambert Wfunction. Solar Energy Materials and Solar Cells. 2004; 81: 269-77.

[5] Saleem H, Karmalkar S. An Analytical Method to Extract the Physical Parameters of a Solar Cell From Four Points on the Illuminated J-V Curve. Electron Device Letters. IEEE. 2009; 30: 349-352.

[6] Chan DSH, Phillips JR, Phang JCH. A comparative study of extraction methods for solar cell model parameters. Solid-State Electronics. 1986; 29: 329-337.

[7] Askarzadeh A, Rezazadeh A. Parameter identification for solar cell models using harmony searchbased algorithms. Solar Energy. 2012; 86: 3241-34249. 
[8] Askarzadeh A, Rezazadeh A. Artificial bee swarm optimization algorithm for parameters identification of solar cell models. Applied Energy. 2013; 102: 943-349.

[9] Wei H, Cong J, Lingyun X. Extracting Solar Cell Model Parameters Based on Chaos Particle Swarm Alogorithm. Electric Information and Control Engineering (ICEICE). 2011 International Conference on 2011: 398-402. doi:10.1109/ICEICE.2011.5777246.

[10] Ishaque K, Salam Z, Mekhilef S, Shamsudin A. Parameter extraction of solar photovoltaic modules using penalty-based differential evolution. Applied Energy. 2012; 99: 297-308.

[11] AlHajri MF, El-Naggar KM, AIRashidi MR, Al-Othman AK. Optimal extraction of solar cell parameters using pattern search. Renewable Energy. 2012; 44: 238-45.

[12] El-Naggar KM, AIRashidi MR, AlHajri MF, Al-Othman AK. Simulated Annealing algorithm for photovoltaic parameters identification. Solar Energy. 2012; 86: 266-74.

[13] Easwarakhanthan T, Bottin J, Bouhouch I, Boutrit C. Nonlinear Minimization Algorithm for Determining the Solar Cell Parameters with Microcomputers. International Journal of Solar Energy. 1986; 4:1-12.

[14] Wolf M, Noel GT, Stirn RJ. Investigation of the double exponential in the current-Voltage characteristics of silicon solar cells. IEEE Transactions on Electron Devices. 1977; 24: 419-28. doi:10.1109/T-ED.1977.18750.

[15] J Nocedal, SJ Wright. Numerical Optimization. 1999.

[16] De Boer PT, Kroese DP, Mannor S, Rubinstein RY. A tutorial on the cross-entropy method. Annals of Operations Research. 2005; 134:19-67.

[17] Dp K. Monte Carlo Methods in Bayesian Computation. Lecture Notes. 2011; 43:1-176.

[18] Rogério dos Santos Alves; Alex Soares de Souza et all. TRATAMIENTOS CRIOGÉNICOS Y LA PROPUESTA DE SU ENSAYO COMO PRÁCTICA DEL LABORATORIO DE METALURGIA Y METALOGRAFÍA. 2014. doi:10.1007/s13398-014-0173-7.2. 